\title{
Wheat straw degradation and production of alternative substrates for nitrogenase of Rhodobacter sphaeroides*
}

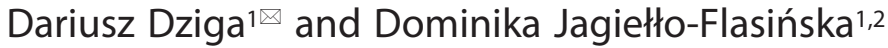 \\ 1Department of Plant Physiology and Development, Faculty of Biochemistry, Biophysics and Biotechnology, Jagiellonian University, Kraków, \\ Poland; 2Department of Plant Biotechnology, Faculty of Biochemistry, Biophysics and Biotechnology, Jagiellonian University, Kraków, Poland
}

Cellulose is a major component of plant biomass and could be applied in the production of biofuels, especially bioethanol. An alternative approach is production of a clean fuel - hydrogen from cellulosic biomass. In this paper an innovatory model of cellulosic waste degradation has been proposed to verify the possibility of utilization of cellulose derivatives by purple non-sulfur bacteria. The concept is based on a two-step process of wheat straw conversion by bacteria in order to obtain an organic acid mixture. In the next stage such products are consumed by Rhodobacter sphaeroides, the known producer of hydrogen. It has been documented that Cellulomonas uda expresses cellulolytic activity in the presence of wheat straw as an only source of carbon. $R$. sphaeroides applied in this research can effectively consume organic acids released from straw by $C$. uda and Lactobacillus rhamnosus and is able to grow in the presence of these substrates. Additionally, an increased nitrogenase activity of $R$. sphaeroides has been indicated when bacteria were cultivated in the presence of cellulose derivatives which suggests that hydrogen production occurs.

Key words: cellulose utilization; cellulose; nitrogenase; purple bacteria

Received: 16 February, 2015; revised: 30 March, 2015; accepted: 17 April, 2015; available on-line: 21 July, 2015

\section{INTRODUCTION}

The generation of clean, sustainable energy from renewable carbon sources (e.g. lignocellulosic biomass, wastewater) has recently been the subject of intensive research and industrial interest. Among different biofuels that may be produced from biomass, hydrogen is considered to be a clean energy carrier. Beside typical photobiological systems which are based on water as a source of electrons and protons (Ghirardi et al., 2009), hydrogen production may occur via dark fermentation of organic wastes or photofermentation that also requires organic material (Basak \& Das, 2007).

Cellulose crop wastes are one of the most attractive sources of organic material which may be used in the production of clean fuel. The most common final product of cellulose utilization is bioethanol, which may be obtained through several different processes; among them consolidated bioprocessing is the most promising option. All the concepts of bioethanol generation from cellulose assume sequential or common action of cellulolytic and ethanogenic microorganisms. However, several disadvantages of ethanol production from cellulose are known. For example, the ability of cellulolytic microorganisms to utilize high concentrations of substrate is questionable (Desvaux et al., 2001). Secondly, growth limitation due to high ethanol concentrations is of particular importance (Olson et al., 2012). An alternative or complementary method of cellulose utilization is fermentative $\mathrm{H}_{2}$ production from this biomass. Direct processes of cellulose hydrolysis followed by hydrogen production offer a limited $\mathrm{H}_{2}$ yield because the reducing sugars are consumed by non-cellulolytic microorganisms. Furthermore, they usually require substrate pretreatment procedures which generate additional production cost. Alternatively, a two-stage process has been demonstrated recently (Lo et al., 2008; Wang et al., 2008; Lo et al., 2009a). In this concept, cellulose is converted to reducing sugars by mixed or pure microbial culture and then hydrogen is produced by using efficient hydrogen producers.

Here, an innovatory model of cellulosic waste utilization is proposed (Fig. 1). It is based on the degradation of wheat straw by cellulolytic bacteria (Cellulomonas uda) followed by the conversion of monosaccharides from cellulosic hydrolyzate by lactic acid bacteria (Lactobacillus rhamnosus) in order to obtain organic acids. In the next stage the organic acid mixture is consumed by the purple bacteria. The main goal of this work was to verify the possibility of utilization of cellulose degradation products

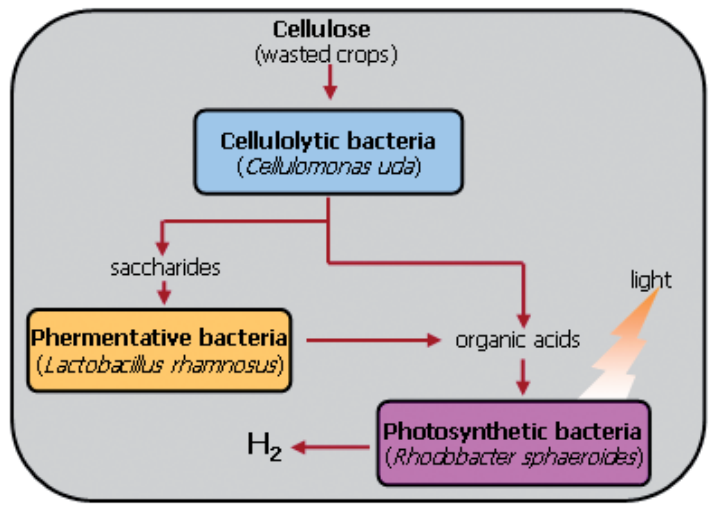

Figure 1. The concept of two- or three-component system of cellulose utilization and hydrogen production.

e-mail: dariusz.dziga@uj.edu.pl

*Presented at 42nd Winter School of the Faculty of Biochemistry, Biophysics and Biotechnology of the Jagiellonian University, February 16-21, 2015, Zakopane, Poland.

Abbreviations: BHM, Bushnell Haas Medium; CMC, carboxymethylcellulose; MRS, de Man, Rogosa, Sharpe medium; MV,- methyl viologen; PNS, purple non-sulphur bacteria 
by Rhodobacter sphaeroides. Additionally, the nitrogenase activity powered by organic substrates derived from cellulose was determined.

\section{MATERIAL AND METHODS}

Materials and bacterial strains Wheat straw as a cellulosic substrate (selected because of their local abundance) was acquired from the local farmers. It was prepared by drying at $105^{\circ} \mathrm{C}$ and grinding to obtain ca. 1 $\mathrm{mm}$ particles.

Cellulolytic bacteria Cellulomonas uda DSMZ 20108 (used in wheat straw hydrolysis) and $\mathrm{H}_{2}$ producer $\mathrm{R} h o$ dobacter sphaeroides DSMZ 5864 (tested as cellulose derivative utilizer) were obtained from the German Collection of Microorganisms and Cell Cultures (Leibniz Institute), whereas Lactobacillus rhamnosus LC 705 able to ferment reduced sugars originating from cellulose was from Valio Ltd. (Helsinki, Finland).

Bacterial cultivation DSMZ 53 medium was used for efficient growth and proliferation of C. uda; the cultivation was carried out at $\mathrm{pH} 7.2-7.4$ in constant temperature of $37^{\circ} \mathrm{C}$ on shaker with speed of $160 \mathrm{rpm}$. After the culture reached a stationary phase $(40 \mathrm{~h})$, the cells were centrifuged and transferred into the BHM (Bushnell Haas Medium) consisting $10 \mathrm{~g} \mathrm{l}^{-1}$ of carboxymethylcellulose (CMC) or ground wheat straw and incubated at $37^{\circ} \mathrm{C}$. L. rhamnosus was cultivated in MRS (de Man, Rogosa, Sharpe) medium at $37^{\circ} \mathrm{C}$ and $6.2-6.5 \mathrm{pH}$. The $\mathrm{R}$. sphaeroides culture was grown using DSMZ 27 medium at $\mathrm{pH} 6.8$ and $20^{\circ} \mathrm{C}$ under illumination of $40 \mathrm{~W}$.

Experimental procedures Determination of cellulolytic activity and wheat straw degradation products. After a 2 day-cultivation of C. uda in DSMZ 53 medium and transferring into BHM medium with wheat straw, cultivation was carried out at $42^{\circ} \mathrm{C}$ on $160 \mathrm{rpm}$ speed shaker. The samples were taken from the culture in the subsequent days and appropriate assays were performed.

Two and three-step models of wheat straw degradation. C. uda cells proliferated in DSMZ 53 medium were centrifuged, washed twice with BHM medium, transferred into BHM medium supplemented with wheat straw and cultivated for an appropriate amount of time (2-5 days, depending on the experiment) at $42^{\circ} \mathrm{C}$ with continuous shaking (160 rpm). Subsequently, after the wheat straw hydrolysis, the cells were centrifuged and the medium was autoclaved and inoculated with L. rhamnosus cells freshly proliferated on MRS medium and washed twice with BHM medium (final density $10^{9}$ cells $\mathrm{ml}^{-1}$ ). The fermentation was performed in falcon tubes at $37^{\circ} \mathrm{C}$ and the samples were collected in the following days of incubation. In a three-step model, L. rhamnosus cells were removed and the medium was sterilized again. R. sphaeroides cells were transferred into such a medium supplemented with microelements (final density $2 \times 10^{7}$ cells $\mathrm{ml}^{-1}$ ) and incubated for the following days at $20^{\circ} \mathrm{C}$ under illumination of $40 \mathrm{~W}$.

Analytical methods The viability of C. uda and $L$. rhamnosus cells was tested on DSMZ 53 and MRS agar plates, respectively. Plates were incubated at $37^{\circ} \mathrm{C}$ for 3 days and then the plate count was determined. The density of PNS culture was determined in a Bürker chamber.

The concentration of reducing sugars released from wheat straw was determined by modified Nelson-Somogyi method. $150 \mu \mathrm{l}$ of tested medium was incubated with $300 \mu \mathrm{l}$ of Nelson-Somogyi reagent at $100^{\circ} \mathrm{C}$ for $15 \mathrm{~min}$. The samples were cooled, mixed with $300 \mu$ l of arsenic molybdenate and gently shaken. Absorbance was measured at a wavelength of $540 \mathrm{~nm}$ using microplate reader iMark (BioRad) with Microplate Manager 6 software.

HPLC method was applied as both a quantitative and qualitative technique to study organic acids. The HPLC equipment used in the research consisted of a pomp Waters 600E Multisolvent Delivery System, an autosampler — Waters 717 plus, a detector — Waters 996 PDA and a thermostat - Jetstream 2 plus with Millenium ${ }^{32}$ SS software. In HPLC separation tests, Nova-Pack C18 column was applied with $5 \mathrm{mM}$ sulfuric acid as a solvent. Separation in isocratic flow was $12 \mathrm{~min}$ at $20^{\circ} \mathrm{C}$

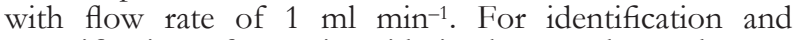
quantification of organic acids in the tested samples, series of standard dilutions were analyzed and their retention times were established.

Enzyme assays. The activity of cellulases secreted by C. $u d a$ was measured by modified colorimetric assays as described by Mielenz et al. (2009) using 96-well plates. The single well which contained a reaction mixture of $100 \mu \mathrm{l}$ of citrate buffer $(50 \mathrm{mM}, \mathrm{pH} 4.8), 100 \mu \mathrm{l}$ of adequate substrate with $100 \mu \mathrm{l}$ of tested medium was incubated at $37^{\circ} \mathrm{C}$ for $5 \mathrm{~h}$. The exogenic substrates for endoglucanases, exoglucanases and xylanases were $1 \%$ CMC, 1\% Avicel and 1\% xylan, respectively. Enzymatic activity was expressed as a level of reducing sugars released from the substrate, indicated colorimetrically with Nelson-Somogyi method using glucose as a standard. $\beta$-glucosidases activity was determined colorimetrically using $p$-nitrophenyl- $\beta$-D-glucuronide as a substrate and the product ( $p$-nitrophenol) was measured at a wavelength of $415 \mathrm{~nm}$.

A simplified assay according to the method of Ren et al. (2005) was applied to indicate the potency of $R$. sphaeroides for hydrogen production. It was assumed (based on the presumption mentioned in the Introduction) that in case of PNS bacteria this assay indicates nitrogenase activity instead of hydrogenase activity. This activity was measured by monitoring the oxidation of reduced methyl viologen (MV) at $604 \mathrm{~nm}$ under illumination of $40 \mathrm{~W}$. The reaction was carried out in a $4-\mathrm{ml}$ glass cuvettes, tightly closed with a cap to prevent ingress of air. The reaction mixture, prepared in an anaerobic chamber contained $4 \mathrm{ml}$ of $0.4 \mathrm{mM} \mathrm{MV}$ in phosphate buffer, $\mathrm{pH} 8,0.1 \%$ Triton $\mathrm{X}-100$ and $50 \mu \mathrm{L}$ of $0.1 \mathrm{M}$ sodium dithionate (which reduces MV efficiently) added directly before the measurement. The cells of $R$. sphaeroides (harvested from the 1-2 week-old culture) previously washed with adequate medium were suspended in media collected after hydrolysis and fermentation of wheat straw (see Experimental procedures) and 0.4 $\mathrm{ml}$ of such a sample was added to the reaction mixture. The final density of the cells in the reaction mixture was $8 \times 10^{7}$ cells $\mathrm{ml}^{-1}$. One unit of nitrogenase activity was defined as the oxidation of $1 \mu \mathrm{mol}$ of reduced MV per min.

Statistical analyses. Statistically significant differences between the xylanase and endoglucanase activity in $\mathrm{BHM} / \mathrm{CMC}$ and $\mathrm{BHM} /$ wheat straw media were found by Anova test. Anova complemented with Tukey test were used to analyze the diversity of nitrogenase activity.

\section{RESULTS}

The cultivation of the C. uda strain in two different media (DSMZ and BHM/CMC) resulted in different growth potential (in $\mathrm{BHM}_{\mathrm{OD}}$ was 10 times lower than the maximum value on DSMZ) and a minimum 

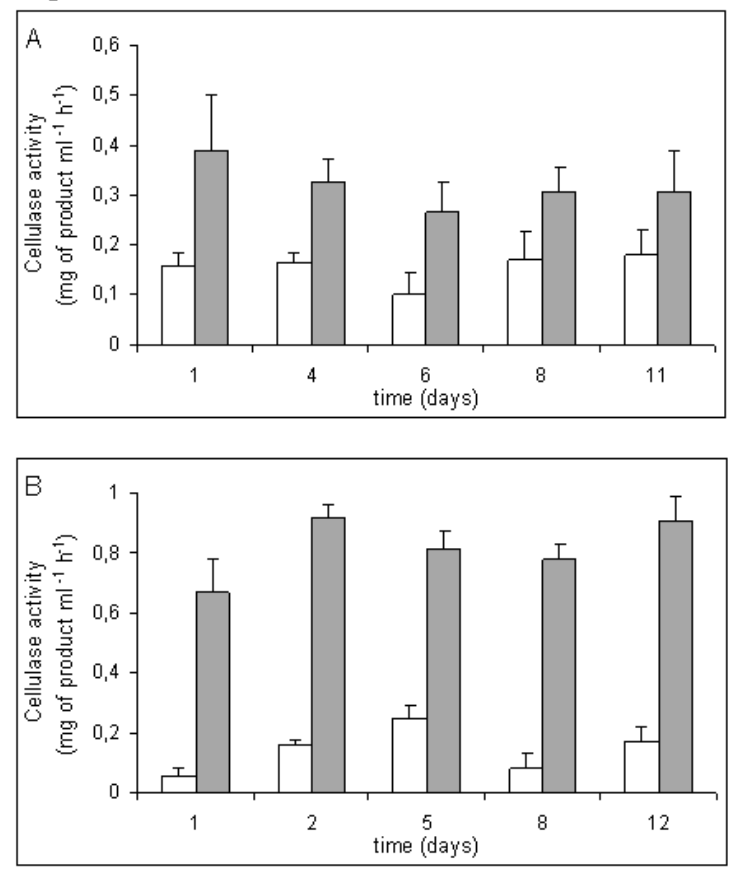

Figure 2. Cellulase activity of $C$. uda during a long-term incubation in BHM/CMC medium (A) and BHM supplemented with wheat straw (B).

A reaction mixture with exogenic substrates (1\% CMC for endoglucanase, $1 \%$ xylan for xylanases) in citrate buffer $(50 \mathrm{mM}, \mathrm{pH}$ 4.8), was incubated at $37^{\circ} \mathrm{C}$ for $5 \mathrm{~h}$. White and gray blocs indicate endoglucanase and xylanase activity, respectively, whereas bars indicate S.D. $(n=3)$. Statistically significant difference of the average xylanase activity between the used media with $p<0.001$ (Anova).

level of cellulase production in both cases (Table 1). However, the cellulolytic activity calculated for the same value of $\mathrm{OD}_{600}$ indicated much higher potential of cellulase production, when the cells were cultivated in BHM/ CMC medium. For this reason a two-stage cultivation model was proposed to maximize the proliferation of bacteria and production of a large quantity of cellulolytic enzymes. In such two-stage procedure the cells quickly proliferated in DSMZ medium and after the transfer to the medium with the $\mathrm{CMC}$ as the sole carbon source, higher production of cellulases was observed (Table 2) when compared to the previous experiment. Cellulase activity was mainly extracellular, except for $\beta$-glucosidase (similarly as in the work by Lo et al. 2009a). In the next experiments, a fast growth of C. uda was obtained by cultivation on DSMZ medium, then the cells were used to hydrolyze the CMC or wheat straw in BHM medium.

Because the highest cellulolytic activity was documented for endoglucanases and xylanases, the next assays

Table 1. Cellulase activity (measured in medium) and optical density of the cultures of $C$. uda after 2 days of cultivation in DSMZ 53 and BHM media.

A reaction mixture with exogenic substrates ( $1 \%$ CMC for endoglucanase, $1 \%$ xylan for xylanases) in citrate buffer $(50 \mathrm{mM}, \mathrm{pH} 4.8)$, was incubated at $37^{\circ} \mathrm{C}$ for $5 \mathrm{~h}$.

\begin{tabular}{|c|c|c|c|c|c|}
\hline \multirow[t]{2}{*}{ Medium } & \multicolumn{2}{|c|}{$\begin{array}{l}\text { Cellulolytic activity } \\
\text { (mg of product } \mathrm{ml}^{-1} \mathrm{~h}^{-1} \text { ) }\end{array}$} & \multirow[t]{2}{*}{$\mathrm{OD}_{600}$} & \multicolumn{2}{|c|}{ 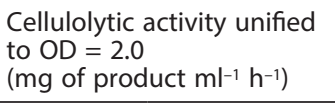 } \\
\hline & xylanase & endoglucanase & & xylanase & endoglucanase \\
\hline DSMZ & 0.011 & 0.018 & 1.944 & 0.005 & 0.010 \\
\hline $\mathrm{BHM} / \mathrm{CMC}$ & 0.012 & 0.012 & 0.186 & 0.710 & 0.673 \\
\hline
\end{tabular}

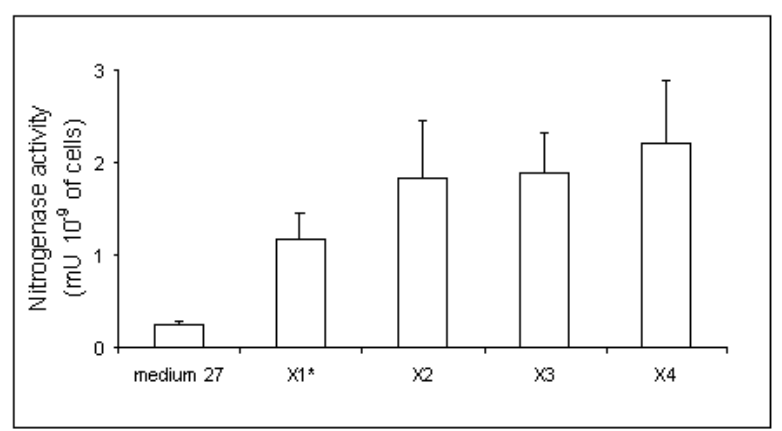

Figure 3. Nitrogenase activity of Rhodobacter sphaeroides in different media: DSMZ 27, 5-day hydrolyzate of wheat straw (X1), hydrolyzate $\mathrm{X} 1$ fermented by L. rhamnosus for 2, 4 and 6 days (X2, X3, X4, respectively).

The oxidation of reduced MV was performed in an anaerobic condition in $\mathrm{pH}$ 8. Error bars indicate standard deviation. Asteriks means significant difference versus $\mathrm{X} 2, \mathrm{X} 3$ and $\mathrm{X} 4$ groups $(\mathrm{F}=$ 8.011, $\mathrm{p}<0.001$ ); (Anova and Tukey test).

were performed only for these two enzymes to monitor the level of activity towards CMC and wheat straw. The long-term activity of endoglucanases and xylanases cultivated in $\mathrm{BHM} / \mathrm{CMC}$ medium indicated a relatively stable level of enzyme production (Fig. 2A). Additionally, the viability of $C$. uda cells during such a 3 -week cultivation was stable (between $1.7 \times 10^{9}$ and $3.4 \times 10^{9}$ colony form units $\mathrm{ml}^{-1}$ ).

A stable level of cellulolytic activity was detected also during long-term incubation of C. uda (previously proliferated in DSMZ medium) in BHM medium supplemented with wheat straw instead of CMC (Fig. 2B). The average endoglucanase activity was similar for both sources of carbon, whereas the average xylanase activity was significantly higher in the medium supplemented with wheat straw. The activity of both enzymes was also comparable with the one observed in the presence of rice straw (data not presented). A colorimetric assay and HPLC analysis of such media after subsequent days of hydrolysis indicated that C. uda hydrolyzed the substrate (wheat straw) producing not only reducing sugars but also organic acids (Table 3). Such hydrolyzate was tested for the possibility of utilization by the L. rhamnosus and R. sphaeroides in a 3-step experiment. As shown in Table 4, 2 days of fermentation of wheat straw hydrolyzate in the presence of $L$. rhamnosus resulted in a decreased amount of reducing sugars and increased level of organic acids. In the next step of wheat straw decomposition the fermentation products were inoculated with $R$. sphaeroides cells which utilized the organic acids effectively. The concentration of organic acids originating from wheat straw decreased very significantly within 4 days of incubation with the cells of PNS bacteria. During this experiment a growth of $R$. sphaeroides was observed, however this growth was slower than in the control (final numbers of cells were $1.8 \times 10^{8}$ and $3.4 \times 10^{8}$ cells $\mathrm{ml}^{-1}$, respectively) which may be a result of far from perfect condition of growth.

To indicate the potency of hydrogen production from cellulose derivatives (mainly organic acids) by R. sphaeroides, a nitrogenase activity assay was performed. Five different samples were analyzed: medium 27 (X0), 5-day hydrolyzate of wheat straw (X1) and the same hydrolyzate after 2, 4 and 6 days of fermentation $\mathrm{X} 2, \mathrm{X} 3, \mathrm{X} 4$, respec- 
Table 2. Cellulase activity of lysate and medium after a two-step cultivation (at $42^{\circ} \mathrm{C}$ on $160 \mathrm{rpm}$ ) of C. uda in DSMZ 53 (48h) and BHM (48h).

A reaction mixture with exogenic substrates (1\% CMC for endoglucanase, 1\% xylan for xylanases) in citrate buffer $(50 \mathrm{mM}, \mathrm{pH} 4.8)$, was incubated at $37^{\circ} \mathrm{C}$ for $5 \mathrm{~h}$.

\begin{tabular}{llccc}
\hline \multirow{2}{*}{$\begin{array}{l}\text { Source of } \\
\text { cellulase }\end{array}$} & \multicolumn{4}{c}{ Cellulose activity $\left(\mathrm{mg}\right.$ of product $\left.\mathrm{ml}^{-1} \mathrm{~h}^{-1}\right)$} \\
\cline { 2 - 5 } & endoglucanase & exoglucanase & B-glucosidase & xylanase \\
\cline { 4 - 5 } cell lysate & 0.004 & 0.025 & 1.109 & 0.194 \\
\hline $\begin{array}{l}\text { medium } \\
\text { BHM/CMC }\end{array}$ & 0.228 & 0.015 & 0.000 & 1.471 \\
\hline
\end{tabular}

tively). The potency of $R$. sphaeroides for hydrogen production (expressed as the ability to oxidize the reduced MV) in the presence of both the wheat straw hydrolyzate and fermentation products was documented (Fig. 3). The nitrogenase activity of $R$. sphaeroides was (in comparison with DSMZ 27 medium) 4.5 times higher in the presence of straw hydrolyzate and 7.1-8.6 times higher in the hydrolyzate after a few days of fermentation. The insignificant differences between groups X2, X3 and X4 may be explained by similar organic acids content in the hydrolysates (see Table 3).

\section{DISCUSSION}

Table 3 . The concentration of selected organic acids and reducing sugars after subsequent days of wheat straw hydrolysis by C. uda (incubation at $42^{\circ} \mathrm{C}$ on $160 \mathrm{rpm}$ ).

\begin{tabular}{|c|c|c|c|}
\hline \multirow{2}{*}{$\begin{array}{l}\text { Time of hydrolysis } \\
\text { (days) }\end{array}$} & \multicolumn{2}{|c|}{ organic acids ( $\mu \mathrm{mol} \mathrm{ml}^{-1}$ ) } & \multirow{2}{*}{$\begin{array}{l}\text { reducing sugars } \\
\left(\mathrm{mg} \mathrm{ml}^{-1}\right)\end{array}$} \\
\hline & acetate & fumarate & \\
\hline 1 & 0.02 & 0.12 & $0.15 \pm 0.03$ \\
\hline 2 & 0.10 & 0.16 & $0.18 \pm 0.04$ \\
\hline 5 & 0.12 & 0.17 & $0.16 \pm 0.04$ \\
\hline 8 & 0.13 & 0.18 & $0.49 \pm 0.59$ \\
\hline 12 & 0.11 & 0.22 & $0.28 \pm 0.26$ \\
\hline
\end{tabular}

In studies of cellulose transformation into biofuels several crucial direction of research may be underlined involving: metabolic engineering to achieve high product yiealds, development of transformation systems, biochemical analyses of the microbial conversion of substrates and designing single or multi-stage systems of biomass utilization and biofuel production. In this paper the biochemical ability of three microbial strains to con2008). vert cellulose and its derivatives was analyzed including both the activity of selected enzymes (cellulases and nitrogenases) and the capability of formation or consumption of subsequent byproducts.

Our idea of three step degradation of wheat straw (Fig. 1) is based on the followed assumptions. Firstly, a reductant for $\mathrm{CO}_{2}$ fixation and nitrogenase activity is provided by the photoassimilation of organic acids. The most common organic acids utilized by PNS bacteria include formate, acetate, lactate, butyrate, etc. (Koku et al., 2002; Asada et al., 2008; Kars et al., 2010). Among the main factors which limit the utilization of the nonoxygenic, nitrogenase-mediated photofermentation process (Akkerman et al., 2002), the availability of sources of organic acids may be crucial. A promising solution to this problem is the production of substrates for PNS using easily available material - cellulose delivered from agricultural residues. Secondly, an efficient conversion of celllulosic derivatives into organic acids can be performed by lactic acid bacteria (Kim et al., 2010; Guo et al., 2010) which are able to metabolize simultaneously both hexoses and pentoses from bran or straw (Givry et al., 2008; Kim et al., 2010) allowing effective utilization of all the sugars released from cellulose.

The fist part of our experiments was performed to confirm the usefulness of wheat straw as a substrate for C. uda strain because the work of Lo et al. (2009b) indicated that C. uda may hydrolyse rice straw effectively. Our documentation of high cellulase activity in the presence of wheat straw confirmed that also this agricultural residue is suitable for the used bacterial strain (Fig. 2). The activity of endoglucanase was comparable to the activity observed in the presence of CMC (present work) and rice straw (Lo et al., 2009b) as a substrate. However, in the presence of CMC the activity of xylanase is on the basic level because no substrate-induced expression of xylanase is possible. On the other hand, it may be concluded that wheat straw induced xylanase production and this may explain significantly higher activity. As was indicated, fast proliferation of $C$. uda cells may be obtained by cultivation in DSMZ medium (Table 1). This approach allows to use a large amount of cellulolytic bacteria for the hydrolysis of cellulose material. On the other hand, such preliminary proliferation of C. uda cells in DSMZ medium generates additional costs. However, such enzymatic degradation of cellulosic feedstock has several advantages over chemical processes like high potential saccharification efficiency, lower energy consumption and limited generation of pollution (Saratale et al.,

Reducing sugars were the main product of wheat straw utilization, however organic acids were also detected in the media after a few days of straw hydrolysis (Table 3). It was assumed that such composition of wheat straw derivatives constitutes a promising substrate for PNS bacteria which can use a wide range of organic compounds like sugars, acids or even waste streams (Eroglu et al., 2004; Uyar et al., 2009). However, among various organic substrates, lactate and malate are suggested to be most promising in hydrogen production whereas acetate and 
glucose are not efficiently converted to hydrogen (Kars et al., 2009). It depends on the substrate assimilation pathway, the redox balance and production of reducing equivalents (Koku et al., 2002). To obtain a more suitable substrate for PNS bacteria, the next step in the cellulose degradation was the production of organic acids from reducing sugars which was performed by cultivation of hydrolyzate in the presence of lactic acid bacteria. These microorganisms have been investigated recently due to a promising conversion efficiency of cellulosic derivatives and effective production of chemicals like lactate or acetate from this feedstock (Kim et al., 2010; Guo et al., 2010; Cui et al., 2011). Furthermore, preferential consumption of sugar, known as carbon catabolite repression may be avoided. As was shown in Table 4, the fermentation of cellulose derivatives by L. rhamnosus strain allowed to produce a higher amount of organic acids (in comparison with hydrolyzate). Among them, the highest concentration of lactic acid was noticed. Additionally, the concentration of reducing sugars was drastically decreased during this process. These results indicated that two-stage decomposition of cellulose - hydrolysis by C. uda and fermentation by. L. rhamnosus - allows to obtain a substrate for hydrogen producing PNS bacteria. It was also demonstrated that such a mixture was effectively utilized by $R$. sphaeroides which consumed organic compounds delivered from cellulose (Table 4) and were allowed to grow under such condition whereas reducing sugars were only slightly utilized.

The disadvantage of nitrogenase-based hydrogen production is that extra energy in the form of ATP is needed in comparison to hydrogenase-based hydrogen production. It requires high photosynthetic activity as well as high input of proton donors. In practice, a large amount of organic substrate must be delivered. Such a source of organic material may be cellulose crop wastes. To the best of our knowledge, only one relatively old paper reported hydrogen production from cellulose by PNS bacteria (in anaerobic coculture with Cellulomonas strain; Odom \& Wall, 1983). A nitrogenase assay performed in our study indicated that these enzymes may be powered by the organic compounds originating from one- or two-stage process of cellulose decomposition. It is interesting that even hydrolysis of wheat straw is enough to enhance the activity of nitrogenase in comparison with cells cultivated in the control medium. However, additional fermentation of hydrolyzate results in a further statistically significant increase of the measured activity (Fig. 3). Nitrogenase is rapidly repressed in the presence of nitrogen compounds like ammonium. Because $\mathrm{NH}_{3}$ is usually a common component of feedstock tested as a source of organic compounds for hydrogen producing PNS strains, it implies a serious challenge. However, among different raw materials tested for this purpose, such as waste water or municipal solid waste, cow dung, whey (Basak \& Das, 2007), the content of nitrogen in straw is very limited. This explains also the higher nitrogenase activity of $R$. sphaeroides measured in straw derivatives in comparison with DSMZ medium 27 which contains yeast extract as a source of nitrogen.

In conclusion, our idea of cellulose utilization by hydrogen producing PNS strain offers several advantages in comparison with other proposals. We documented that the cellulose derivatives (created by one- or two-stage degradation by C. uda and L. rhamnosus) are consumed by $R$. sphaeroides and may enhance the nitrogenase activity. This promising finding needs intensive further research, including an optimisation of organic acid production from straw, characterization of metabolic pathways of these compounds in R. sphaeroides cells and determination of the efficiency of hydrogen production based on the utilization of wheat straw derivatives.

\section{Acknowledgements}

The authors gratefully acknowledge Prof. Seppo Salminen, University of Turku, Finland for providing the $L$. rhamnosus LC 705 strain.

Faculty of Biochemistry, Biophysics and Biotechnology of Jagiellonian University is a partner of the Leading National Research Center (KNOW) supported by the Ministry of Science and Higher Education.

\section{REFERENCES}

Akkerman I, Janssen M, Rocha J, Wijffels RH (2002) Photobiological hydrogen production: Photochemical efficiency and bioreactor design. Int J Hydrogen Energ 27: 1195-1208.

Asada Y, Ohsawa M, Nagai Y, Ishimi K, Fukatsu M, Hideno A, Wakayama T, Miyake J (2008) Re-evaluation of hydrogen productivity from acetate by some photosynthetic bacteria. Int J Hydrogen Energ 33: $5147-5150$.

Basak N, Das D (2007) The prospect of purple non-sulfur (PNS) photosynthetic bacteria for hydrogen production: the present state of the art. World J Microb Biot 23: 31-42.

Cui F, Yebo L, Wan C (2011) Lactic acid production from corn stover using mixed cultures of Lactobacillus rhamnosus and Lactobacillus brevis. Bioresource Technol 102: 1831-1836.

Desvaux M, Guedon E, Petitdemange H (2001) Kinetics and metabolism of cellulose degradation at high substrate concentrations in steady-state continuous cultures of Clostridium cellulolyticum on a chemically defined medium. Appl Environ Microbiol 67: 3837-3845.

Eroglu E, Gunduz U, Yucel M, Turker L, Eroglu, I (2004) Photobiological hydrogen production by using olive mill wastewater as a sole substrate source. Int J Hydrogen Energ 29: 163-171.

Ghirardi ML, Dubini A, Yu J, Maness PC (2009) Photobiological hydrogen-producing systems Chem Soc Rev 38: 52-61.

Givry S, Prevot V, Duchiron F (2008) Lactic acid production from hemicellulosic hydrolyzate by cells of Lactobacillus bifermentans immobilized in Ca-alginate using response surface methodology. World J Microb Biot 24: 745-752.

Guo W, Wendi J, Li Y, Chen S (2010) Performances of Lactobacillus brevis for producing lactic acid from hydrolysate of lignocellulosics. Appl Biochem Biotech 161: 124-136.

Kars G, Gunduz U, Yucel M, Rakhely G, Kovacs K, Eroglu I (2009) Evaluation of hydrogen production by Rhodobacter sphaeroides O.U.001 and its hupSL deficient mutant using acetate and malate as carbon sources. Int J Hydrogen Energ 34: 2184-2190.

Kars G, Gunduz, U (2010) Towards a super $\mathrm{H}_{2}$ producer: Improvements in photofermentative biohydrogen production by genetic manipulations. Int J Hydrogen Energ 35: 664-656.

Kim JH, Block DE, Mills DA (2010) Simultaneous consumption of pentose and hexose sugars: an optimal microbial phenotype for efficient fermentation of lignocellulosic biomass. Appl Microbiol Biot 88: 1077-1085.

Koku H, Eroglu I, Gunduz U, Yucel M, Turker L (2002) Aspects of the metabolism of hydrogen production by Rhodobacter sphaeroides. Int J Hydrogen Energ 27: 1315-1329.

Lo YC, Bai MD, Chen WM, Chang JS (2008) Cellulosic hydrogen production with a sequencing bacterial hydrolysis and dark fermentation strategy. Bioresource Technol 99: 8299-8303.

Lo YC, Su YC, Chen CY, Chen WM, Lee KS, Chang JS (2009a) Biohydrogen production from cellulosic hydrolysate produced via temperature-shift-enhanced bacterial cellulose hydrolysis. Bioresource Technol 100: 5802-5807.

Lo YC, Saratale GD, Chen WM, Baic MD, Chang JS (2009b) Isolation of cellulosehydrolytic bacteria and applications of the cellulolytic enzymes for cellulosic biohydrogen production. Enzyme Microb Tech 44: $417-425$.

Odom JM, Wall JD (1983) Photoproduction of $\mathrm{H}_{2}$ from cellulose by an anaerobic bacterial coculture. App Environ Microbiol 45: $1300-$ 1305.

Olson DG, McBride JE, Shaw AJ, Lynd LR (2012) Recent progress in consolidated bioprocessing. Curr Opin Biotech 23: 396-405.

Ren, YL, Xing XH, Zhang Ch, Gou ZX (2005) A simplified method for assay of hydrogenase activities of $\mathrm{H}_{2}$ evolution and uptake in Enterobacter aerogenes. Biotechnol Lett 27: 1029-1033.

Saratale GD, Chen SD, Lo YC, Saratale RG, Chang JS (2008) Outlook of biohydrogen production from lignocellulosic feedstock using dark fermentation — a review. J Sci Ind Res 67: 962-979. 
Uyar B, Eroglu I, Yucel M, Gunduz U (2009) Photofermentative hydrogen production from volatile fatty acids present in dark fermentation effluents. Int J Hydrogen Energ 34: 4517-4523.

Wang A, Ren N, Shi Y, Lee DJ (2008) Bioaugmented hydrogen production from microcrystalline cellulose using co-culture Clostridium acetobutylicum X9 and Ethanoigenens harbinense B49. Int I Hydrogen Energ 33: 912-917.

Zhang YHP, Hong J, Ye Z (2009) Cellulase assays. In Biofuels. Methods and protocols. Mielenz RJ, ed, pp 213-232, Humana Press. 